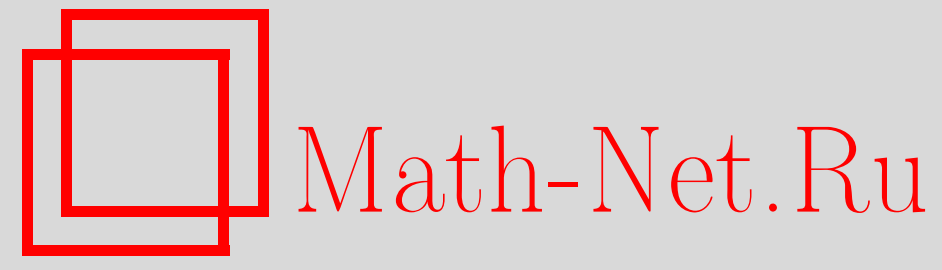

И. А. Кареев, Нижние границы для среднего объема выборки и эффективность последовательных процедур упорядочивания, Теория вероятн. и ее примен., 2013, том 58, выпуск $3,591-597$

DOI: https://doi.org/10.4213/tvp4529

Использование Общероссийского математического портала Math-Net.Ru подразумевает, что вы прочитали и согласны с пользовательским соглашением http: //www . mathnet.ru/rus/agreement

Параметры загрузки:

IP : 52.87 .193 .239

26 апреля 2023 г., 12:42:28

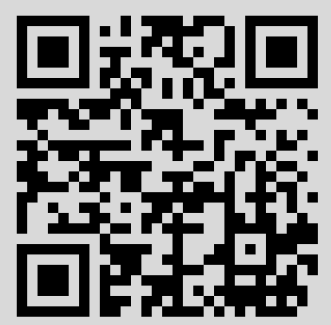


(C) $2013 \Gamma$.

КАРЕЕВ И. А.*

\title{
НИЖНИЕ ГРАНИЦЫ ДЛЯ СРЕДНЕГО ОБЪЕМА ВЫБОРКИ И ЭФФЕКТИВНОСТЬ ПОСЛЕДОВАТЕЛЬНЫХ ПРОЦЕДУР УПОРЯДОЧИВАНИЯ
}

\begin{abstract}
Устанавливаются некоторые нижние границы для среднего объсма выборки в последовательных процедурах упорядочивания популяций по возрастанию значения скалярного параметра, когда гарантируется заданная вероятность корректного решения. Аналогичные границы для последовательных гарантийных процедур отбора были получены в статье автора [3]. При построении границ используются универсальные границы Володина-Малютова [1], [4]. Полученные результаты применяются для оценки эффективности последовательного варианта процедуры упорядочивания Бекхофера [6].
\end{abstract}

Ключевые слова и фразы: упорядочивания популяций, нижние границы для среднего объема выборки, эффективность.

1. Введение. Рассматривается задача упорядочивания $m$ популяций по значениям скалярного параметра $\theta$. При этом предполагается, что распределения наблюдаемых случайных характеристик популяций принадлежат единому семейству распределений, индексируемых скалярным параметром $\theta$. Заметим, что задача упорядочивания связана с задачей отбора, что приводит к схожим принципам их решения.

Задача упорядочивания популяций впервые была поставлена в работе Р. Бекхофера [5]. В этой же статье представлена и процедура упорядочивания Бекхофера с зоной безразличия, решающая задачу для случая нормальной модели с известной и одинаковой дисперсией. Последовательный вариант этой процедуры упорядочивания популяций из семейств экспоненциальных распределений Купмена-Дармуа представлен в монографии [6]. Ряд процедур упорядочивания, в частности по масштабному параметру, для широкого класса распределений представлены в статьях [10], [8]. В [7] предложена двухступенчатая процедура упорядочивания нормальной популяции по средним значениям при неизвестных дисперсиях и приведены примеры ее применения к реальным данным. Проблема использования различных функций потерь обсуждается в [11].

В данной работе рассматриваются последовательные процедуры упорядочивания с введением зоны безразличия (подход Бекхофера), без которой вряд ли возможно построение гарантийных процедур с конечным числом наблюдений. Различные применения этого подхода к построению гарантийных процедур упорядочивания и отбора, как основанных на фиксированном числе наблюдений, так и последовательных, можно найти в монографии [6].

Одной из важнейших характеристик гарантийных процедур является средний объем выборки. Нижние границы позволяют оценить снизу средний объем выборки для всевозможных гарантийных процедур упорядочивания. Кроме того что такая граница представляет интерес сама по себе - как некоторый ориентир при планировании объема наблюдений, она также может использоваться для оценки эффективности процедур упорядочивания, т.е. позволяет оценить, насколько средний объем наблюдений конкретной процедуры далек от возможного минимума.

Целью этой работы является построение нижних границ для среднего объема выборки последовательных гарантийных процедур упорядочивания и применение этих

* Казанский федеральный университет, Казань, Россия; e-mail: kareevia@ gmail.com 
границ для проверки эффективности процедур упорядочивания. При построении границ используются, как и в предыдущей статье [3], универсальные нижние границы для среднего объема выборки Володина-Малютова [1], [2], [4], [9]. Второй пункт посвящен выводу нижних границ посредством применения несколько видоизмененного метода Гальчука-Малютова [9] при решении задач на минимакс. В третьем пункте мы переходим непосредственно к вычислению границ для нормальных и показательных распределений. В четвертом пункте полученные границы применяются к исследованию эффективности последовательной процедуры упорядочивания Бекхофера [6] в нормальной модели с общей известной дисперсией.

2. Нижняя граница для среднего объема выборки. Рассматривается задача упорядочивания $m$ популяций по возрастанию значений параметра $\theta \in \Theta \subseteq \mathbf{R}^{1}$. Предполагается, что наблюдаемая случайная характеристика $\xi_{i}$, соответствующая $i$ й популяции, имеет распределение $\mathbf{P}_{\theta_{i}} \in \mathscr{P}=\left\{\mathbf{P}_{\theta}, \theta \in \Theta\right\}$ со значением параметра $\theta=\theta_{i}, i=1, \ldots, m$. В эксперименте наблюдаются независимые копии случайных величин $\xi_{1}, \ldots, \xi_{m}$. Этой задаче соответствует пространство решений $\mathscr{D}$, содержащее $m$ ! элементов - всевозможные перестановки индексов $1, \ldots, m$.

Пусть $\theta_{[1]} \leqslant \cdots \leqslant \theta_{[m]}$ - упорядоченные значения $\theta$ в популяциях.

Далее будут рассматриваться процедуры упорядочивания с зоной безразличия. Введение зоны безразличия состоит в сужении исходного параметрического пространства $\Theta^{m}$ до некоторого пространства $\Theta_{\Delta}(\Delta>0)$, где фиксированное значение $\Delta$ определяет размеры зоны безразличия. Заданное ограничение $1-\alpha(>1 / 2)$ на вероятность корректного решения гарантируется только на $\Theta_{\Delta}$.

Для общей проблемы упорядочивания предлагается ввести зону безразличия следующим образом. Определим функцию $r_{\Delta}(\theta), \Delta>0, \theta \in \Theta$, удовлетворяющую следующим условиям: $r_{\Delta}(\theta)$ строго возрастает по $\theta$ при каждом фиксированном $\Delta$, строго убывает по $\Delta$ при каждом фиксированном $\theta$ и $r_{\Delta}(\theta)<\theta$. Параметрическое пространство с зоной безразличия определим формулой

$$
\Theta_{\Delta}=\left\{\boldsymbol{\theta} \in \Theta^{m}: \theta_{[i]} \leqslant r_{\Delta}\left(\theta_{[i+1]}\right), 1 \leqslant i \leqslant m-1\right\} .
$$

Например, когда $\theta$ - параметр сдвига, естественно положить $r_{\Delta}(\theta)=\theta-\Delta$, чему соответствует

$$
\Theta_{\Delta}=\left\{\boldsymbol{\theta} \in \Theta^{m}: \theta_{[i+1]}-\theta_{[i]} \geqslant \Delta, 1 \leqslant i \leqslant m-1\right\},
$$

а в случае параметра масштаба полагаем $r_{\Delta}(\theta)=(1-\Delta) \theta$, так что

$$
\Theta_{\Delta}=\left\{\boldsymbol{\theta} \in \Theta^{m}: \frac{\theta_{[i]}}{\theta_{[i+1]}} \leqslant 1-\Delta, 1 \leqslant i \leqslant m-1\right\} .
$$

Введем подмножество пространства $\Theta_{\Delta}$

$$
B_{\Delta}(\boldsymbol{\theta})=\left\{\boldsymbol{\vartheta} \in \Theta_{\Delta}: D_{\boldsymbol{\theta}} \cap D_{\boldsymbol{\vartheta}}=\varnothing\right\},
$$

где $D_{\boldsymbol{\theta}} \subseteq \mathscr{D}$ - подмножество корректных для $\boldsymbol{\theta}$ решений. Пусть $I\left(\theta_{i}, \vartheta_{i}\right)$ - различающая информация по Кульбаку-Лейблеру между распределениями $\mathbf{P}_{\theta_{i}}$ и $\mathbf{P}_{\vartheta_{i}}$.

Используя универсальные границы Володина-Малютова, представим нижнюю границу для среднего значения $\mathbf{E}_{\boldsymbol{\theta}} \nu$ общего для всех популяций объема наблюдений в виде

$$
\mathbf{E}_{\boldsymbol{\theta}} \nu \geqslant \inf _{\mathbf{w} \in W} \sup _{\boldsymbol{\vartheta} \in B_{\Delta}(\boldsymbol{\theta})} \frac{\omega(\alpha(\boldsymbol{\theta}), \alpha(\boldsymbol{\vartheta}))}{\sum_{i=1}^{m} w_{i} I\left(\theta_{i}, \vartheta_{i}\right)}, \quad \boldsymbol{\theta} \in \Theta_{\Delta}
$$

где

$$
\omega(x, y)=x \ln \frac{x}{1-y}+(1-x) \ln \frac{1-x}{y}
$$

- функция Вальда, $\alpha(\boldsymbol{\theta})$ — вероятность ошибочного решения процедуры упорядочивания и 


$$
W=\left\{\mathbf{w} \in[0 ; 1]^{m}: \sum_{i=1}^{m} w_{i}=1\right\}
$$

- единичный симплекс.

Заметим, что $\omega(x, y)$ убывает по $x$ и по $y$ при $x+y<1$. Поэтому для заданного ограничения $\alpha$ на вероятность ошибочного решения $\alpha(\boldsymbol{\theta}) \leqslant \alpha$ получаем нижнюю границу для гарантийной процедуры отбора:

$$
\mathbf{E}_{\boldsymbol{\theta}} \nu \geqslant \frac{\omega(\alpha, \alpha)}{\sup _{\mathbf{w} \in W} \inf _{\boldsymbol{\vartheta} \in B_{\Delta}(\boldsymbol{\theta})} \sum_{i=1}^{m} w_{i} I\left(\theta_{i}, \vartheta_{i}\right)} .
$$

В дальнейшем различающая информация $I(u, v), u, v \in \Theta \subset \mathbf{R}^{1}$, будет называться строго монотонной, если при каждом фиксированном $u$ информация $I(\cdot, v)$ как функция от $v$ убывает при $v<u$ и возрастает при $v>u$. Для устранения громоздкости формул квадратные скобки в записи упорядоченных значений вектора $\theta$ будут опускаться, так что всегда $\theta_{1} \leqslant \cdots \leqslant \theta_{m}$.

Введём для $1 \leqslant i \leqslant m-1$ множества $B_{\Delta}^{i}(\boldsymbol{\theta})$, являющиеся ключевыми при формулировке основного результата работы. Пусть $B_{\Delta}^{i}(\boldsymbol{\theta})$ - это множество тех значений $\vartheta \in\left[\theta_{i} ; r_{\Delta}^{-1}\left(\theta_{i+1}\right)\right]$, для которых выполняется условие

$$
\left(\theta_{1}, \ldots, \theta_{i-1}, \vartheta, r_{\Delta}(\vartheta), \theta_{i+2}, \ldots, \theta_{m}\right) \in B_{\Delta}(\boldsymbol{\theta}) .
$$

Например, для случая параметра сдвига, когда $m=2$ и $\Theta=\mathbf{R}^{1}$, имеем $B_{\Delta}^{1}(\boldsymbol{\theta})=$ $\left[\theta_{1} ; \theta_{2}+\Delta\right]$. Если $m=3$, то

$$
B_{\Delta}^{1}(\boldsymbol{\theta})=\left[\theta_{1} ; \min \left\{\theta_{3}-\Delta, \theta_{2}+\Delta\right\}\right], \quad B_{\Delta}^{2}(\boldsymbol{\theta})=\left[\max \left\{\theta_{1}+2 \Delta, \theta_{2}\right\} ; \theta_{3}+\Delta\right] .
$$

Теорема 1. Пусть семейство распределений $\mathscr{P}$ таково, что различающая информачия $I(u, v)$ строго монотонна. Тогда для любого $\boldsymbol{\theta} \in \Theta_{\Delta}$ справедливь оченки:

(a) если $m=2$, mo

$$
\mathbf{E}_{\boldsymbol{\theta}} \nu \geqslant \frac{\omega(\alpha, \alpha)}{\sup _{w \in[0 ; 1]} \inf _{\vartheta \in B_{\Delta}^{1}(\boldsymbol{\theta})}\left(w I\left(\theta_{1}, \vartheta\right)+(1-w) I\left(\theta_{2}, r_{\Delta}(\vartheta)\right)\right)} ;
$$

(b) если $m \geqslant 3, m o$

$$
\mathbf{E}_{\boldsymbol{\theta}} \nu \geqslant \sum_{i=1}^{m-1} \frac{\omega(\alpha, \alpha)}{V_{i}}
$$

где

$$
\begin{aligned}
V_{1} & =\sup _{w \in[0 ; 1]} \inf _{\vartheta \in B_{\Delta}^{1}(\boldsymbol{\theta})}\left(w I\left(\theta_{1}, \vartheta\right)+2(1-w) I\left(\theta_{2}, r_{\Delta}(\vartheta)\right)\right), \\
V_{i} & =\sup _{w \in[0 ; 1]} \inf _{\vartheta \in B_{\Delta}^{i}(\boldsymbol{\theta})} 2\left(w I\left(\theta_{i}, \vartheta\right)+(1-w) I\left(\theta_{i+1}, r_{\Delta}(\vartheta)\right)\right), \\
V_{m-1} & =\sup _{w \in[0 ; 1]} \inf _{\vartheta \in B_{\Delta}^{m-1}(\boldsymbol{\theta})}\left(2 w I\left(\theta_{m-1}, \vartheta\right)+(1-w) I\left(\theta_{m}, r_{\Delta}(\vartheta)\right)\right)
\end{aligned}
$$

nри $2 \leqslant i \leqslant m-2$.

Идея доказательства этой теоремы в целом аналогична доказательству теоремы 1 в [3]. Поэтому для краткости доказательство будет опущено.

3. Нижняя граница для нормальных популяций. Пусть $\xi_{i} \sim \mathscr{N}\left(\theta_{i}, \sigma^{2}\right)$, где дисперсия $\sigma^{2}$ известна. Рассматривается задача упорядочивания популяций по возрастанию среднего $\theta_{i} \in \Theta=\mathbf{R}^{1}$. Определим функцию $r_{\Delta}(\theta)=\theta-\Delta$, чему соответствует

$$
\Theta_{\Delta}=\left\{\boldsymbol{\theta} \in \Theta^{m}: \theta_{i+1}-\theta_{i} \geqslant \Delta, 1 \leqslant i \leqslant m-1\right\} .
$$

Для нормальной модели различающая информация принимает вид

$$
I(\theta, \vartheta)=\frac{(\theta-\vartheta)^{2}}{2 \sigma^{2}} .
$$


Предложение 1. Для среднего объема выборки прочедуры упорядочивания нормальной модели для $\boldsymbol{\theta} \in \Theta_{\Delta}$ верны оченки:

(a) если $m=2$, mo

$$
\mathbf{E}_{\boldsymbol{\theta}} \nu \frac{8 \sigma^{2} w(\alpha, \alpha)}{\left(\theta_{2}-\theta_{1}+\Delta\right)^{2}}
$$

(b) если $m \geqslant 3$, mo

$$
\mathbf{E}_{\boldsymbol{\theta}} \nu \geqslant \sum_{i=1}^{m-1} \frac{4 \sigma^{2} w(\alpha, \alpha)}{\left(\theta_{i+1}-\theta_{i}+\Delta\right)^{2}} .
$$

Д о к а з а т е л ь с т в о. Очевидно, что условие строгой монотонности различающей информации выполняется для рассматриваемой нормальной модели, а значит, возможно применение теоремы 1.

Покажем, что

$$
\begin{gathered}
\sup _{w \in[0 ; 1]} \inf _{\vartheta \in\left[\theta_{i}, \theta_{i+1}+\Delta\right]}\left(w \frac{\left(\theta_{i}-\vartheta\right)^{2}}{2 \sigma^{2}}+(1-w) \frac{\left(\theta_{i+1}-\vartheta+\Delta\right)^{2}}{2 \sigma^{2}}\right) \\
=\frac{\left(\theta_{i+1}-\theta_{i}+\Delta\right)^{2}}{8 \sigma^{2}} .
\end{gathered}
$$

Найдем точку $\vartheta_{i}^{*} \in\left[\theta_{i} ; \theta_{i+1}+\Delta\right]$ из условия $\left(\theta_{i}-\vartheta_{i}^{*}\right)^{2} /\left(2 \sigma^{2}\right)=\left(\theta_{i+1}-\vartheta_{i}^{*}+\Delta\right)^{2} /\left(2 \sigma^{2}\right)$ :

$$
\vartheta_{i}^{*}=\frac{\theta_{i}+\theta_{i+1}+\Delta}{2} .
$$

Исходя из этих условий, несложно показать, что левая часть (2) равна

$$
\frac{1}{\sigma^{2}}\left(\theta_{i}-\vartheta^{*}\right)^{2}=\frac{\left(\theta_{i+1}-\theta_{i}+\Delta\right)^{2}}{8 \sigma^{2}}
$$

т.е. равенство (2) выполняется.

Легко видеть, что доказываемая нижняя граница в случае $m=2$ немедленно следует из теоремы 1 и равенства (2).

Рассмотрим теперь случай $m \geqslant 3$. Для получения доказываемой нижней границы нам необходимо лишь оценить величины $V_{i}$ из теоремы 1 . Очевидно, что $\vartheta_{i}^{*} \in B_{\Delta}^{i}(\boldsymbol{\theta})$ при любых $\Delta$ и $\boldsymbol{\theta}$. Таким образом, из (2) получаем

$$
V_{i} \leqslant \frac{\left(\theta_{i+1}-\theta_{i}+\Delta\right)^{2}}{4 \sigma^{2}}, \quad 1 \leqslant i \leqslant m-1,
$$

со знаком равенства при $2 \leqslant i \leqslant m-2$. Предложение 1 доказано.

4. Нижняя граница для показательных популяций. Пусть $\xi_{i} \sim E\left(\theta_{i}\right)$ с функцией плотности $f\left(x \mid \theta_{i}\right)=\exp \left\{-x / \theta_{i}\right\} / \theta_{i}, x \geqslant 0$. Рассматривается задача упорядочения популяций по возрастанию среднего $\theta_{i} \in \Theta, \Theta=(0,+\infty)$. Введем функцию $r_{\Delta}(\theta)=(1-\Delta) \theta$, чему соответствует

$$
\Theta_{\Delta}=\left\{\boldsymbol{\theta} \in \Theta^{m}: \frac{\theta_{i}}{\theta_{i+1}} \leqslant 1-\Delta, 1 \leqslant i \leqslant m-1\right\} .
$$

Для показательной модели различающая информация имеет вид

$$
I(\theta, \vartheta)=\frac{\theta}{\vartheta}-\ln \frac{\theta}{\vartheta}-1 .
$$

Предложение 2. Для среднего объема выборки прочедуры упорядочивания показательной модели при любом $\boldsymbol{\theta} \in \Theta_{\Delta}$ верны оченки:

(a) если $m=2$, mо

$$
\mathbf{E}_{\boldsymbol{\theta}} \nu \geqslant \frac{w(\alpha, \alpha)}{\theta_{2} /((1-\Delta) \vartheta)-\ln \left\{\theta_{2} /((1-\Delta) \vartheta)\right\}-1},
$$


гдe

$$
\vartheta=\frac{\theta_{2} /(1-\Delta)-\theta_{1}}{\ln \left\{\theta_{2} /(1-\Delta)\right\}-\ln \theta_{1}}
$$

(b) если $m \geqslant 3$, mo

$$
\mathbf{E}_{\boldsymbol{\theta}} \nu \geqslant \frac{1}{2} \sum_{i=1}^{m-1} \frac{w(\alpha, \alpha)}{\theta_{i+1} /\left((1-\Delta) \vartheta_{i}\right)-\ln \left\{\theta_{i+1} /\left((1-\Delta) \vartheta_{i}\right)\right\}-1},
$$

где

$$
\begin{gathered}
\vartheta_{i}=\min \left\{(1-\Delta) \theta_{i+2}, \frac{\theta_{i+1} /(1-\Delta)-\theta_{i}}{\ln \left\{\theta_{i+1} /(1-\Delta)\right\}-\ln \theta_{i}}\right\}, \quad 1 \leqslant i \leqslant m-2, \\
\vartheta_{m-1}=\frac{\theta_{m} /(1-\Delta)-\theta_{m-1}}{\ln \left\{\theta_{m} /(1-\Delta)\right\}-\ln \theta_{m-1}} .
\end{gathered}
$$

Д о к а з а т е л ь с т в о. Рассмотрим сначала случай $m \geqslant 3$. Легко увидеть, что условие строгой монотонности различающей информации теоремы 1 выполняется. Поэтому нам остаётся лишь оценить значения $V_{i}$. Для начала оценим величину

$$
\begin{aligned}
2 \sup _{w \in[0 ; 1]} & \inf _{\vartheta \in\left[\theta_{i} ; \theta_{i+1} /(1-\Delta)\right]}\left(w\left(\frac{\theta_{i}}{\vartheta}-\ln \frac{\theta_{i}}{\vartheta}-1\right)\right. \\
& \left.+(1-w)\left(\frac{\theta_{i+1}}{(1-\Delta) \vartheta}-\ln \frac{\theta_{i+1}}{(1-\Delta) \vartheta}-1\right)\right)
\end{aligned}
$$

где $1 \leqslant i \leqslant m-1$. При фиксированном $w$ инфимум по $\vartheta$ в (3) достигается в точке $\vartheta=w \theta_{i}+(1-w) \theta_{i+1} /(1-\Delta)$, что является непрерывной относительно $w$ функцией. Отсюда несложно вывести, что (3) равно

$$
2\left(\frac{\theta_{i+1}}{(1-\Delta) \vartheta_{i}^{*}}-\ln \frac{\theta_{i+1}}{(1-\Delta) \vartheta_{i}^{*}}-1\right)
$$

где $\vartheta_{i}^{*}$ находится из условия

$$
\frac{\theta_{i}}{\vartheta_{i}^{*}}-\ln \frac{\theta_{i}}{\vartheta_{i}^{*}}-1=\frac{\theta_{i+1}}{(1-\Delta) \vartheta_{i}^{*}}-\ln \frac{\theta_{i+1}}{(1-\Delta) \vartheta_{i}^{*}}-1
$$

и имеет вид

$$
\vartheta_{i}^{*}=\frac{\theta_{i+1} /(1-\Delta)-\theta_{i}}{\ln \left\{\theta_{i+1} /(1-\Delta)\right\}-\ln \theta_{i}} .
$$
то

Покажем, что $(1-\Delta) \vartheta_{i}^{*} / \theta_{i} \geqslant 1$. Так как $x$ растёт быстрее, чем $\ln x$, при $x>1$,

$$
\frac{1-\Delta}{\theta_{i}} \vartheta_{i}^{*} \geqslant \frac{(1-\Delta)^{-1}-(1-\Delta)}{-2 \ln (1-\Delta)}
$$

Дифференцируя по $\Delta$ числитель и знаменатель последней дроби, легко находим, что числитель больше знаменателя для любого $\Delta>0$. Следовательно, $\theta_{i} \leqslant(1-\Delta) \vartheta_{i}^{*}$, а значит,

$$
\begin{gathered}
\vartheta_{i}=\min \left\{(1-\Delta) \theta_{i+2}, \vartheta_{i}^{*}\right\} \in B_{\Delta}^{i}(\boldsymbol{\theta}), \quad 1 \leqslant i \leqslant m-2, \\
\vartheta_{m-1}=\vartheta_{m-1}^{*} \in B_{\Delta}^{m-1}(\boldsymbol{\theta}) .
\end{gathered}
$$

Остается заметить, что так как точка $\vartheta_{i}^{*}$ выбиралась из условия $I\left(\theta_{i}, \vartheta_{i}^{*}\right)=$ $I\left(\theta_{i+1},(1-\Delta) \vartheta_{i}^{*}\right)$ и $\vartheta_{i} \leqslant \vartheta_{i}^{*}$, то

$$
\sup _{w \in[0 ; 1]}\left(w I\left(\theta_{i}, \vartheta_{i}\right)+(1-w) I\left(\theta_{i+1},(1-\Delta) \vartheta_{i}\right)\right)=I\left(\theta_{i+1},(1-\Delta) \vartheta_{i}\right) .
$$


Таким образом,

$$
V_{i} \leqslant 2\left(\frac{\theta_{i+1}}{(1-\Delta) \vartheta_{i}}-\ln \frac{\theta_{i+1}}{(1-\Delta) \vartheta_{i}}-1\right), \quad 1 \leqslant i \leqslant m-1 .
$$

Рассмотрим случай $m=2$. Используя найденное значение для (3) и теорему 1 , легко получаем доказываемую нижнюю границу. Предложение 2 доказано.

5. Эффективность последовательной процедуры упорядочивания Бекхофера для нормального случая. Исследуем асимптотическую $(\alpha \rightarrow 0)$ эффективность последовательной процедуры упорядочивания Бекхофера [6] для задачи упорядочивания популяций с нормальным распределением по значениям среднего при общей известной дисперсии. Под асимптотической эффективностью будем понимать величину

$$
\mathscr{E}(\boldsymbol{\theta})=\lim _{\alpha \rightarrow 0} \frac{\inf _{\psi \in H(\alpha)} \mathbf{E}_{\boldsymbol{\theta}} \nu_{\psi}}{\mathbf{E}_{\boldsymbol{\theta}} \nu_{\varphi}}
$$

где $\varphi$ - процедура упорядочивания, эффективность которой рассматривается, а $H(\alpha)$ - множество всех процедур, вероятность корректного упорядочивания в которых не меньше $1-\alpha$ для любого $\boldsymbol{\theta} \in \Theta_{\Delta}$.

Сразу заметим, что для нормальной модели наихудшим для упорядочивания случаем является ситуация, когда $\theta_{i+1}-\theta_{i}=\Delta, 1 \leqslant i \leqslant m-1$, т.е. когда популяции похожи настолько, насколько это возможно в рамках параметрического пространства с зоной безразличия.

Очевидно, что выполняется следующая лемма.

Лемма 1. Верна асимптотическая формула

$$
\omega(\alpha, \alpha)=-\ln \alpha+o(1), \quad \alpha \rightarrow 0 .
$$

Перейдем теперь к рассмотрению эффективности последовательной процедуры упорядочивания Бекхофера [6]. Эта процедура обладает достаточно простым управлением: на каждом шаге наблюдается по одному значению из каждой популяции и после этого решается вопрос об остановке или продолжении эксперимента. Для среднего объема выборки последовательной процедуры Бекхофера верна асимптотическая $(\alpha \rightarrow 0)$ формула (см. [6, раздел 6.4]):

$$
\mathbf{E}_{\boldsymbol{\theta}} \nu=-\frac{m \ln \alpha}{\Delta \min _{1 \leqslant i \leqslant m-1}\left(\theta_{i+1}-\theta_{i}\right)}(1+O(1)), \quad \alpha \rightarrow 0 .
$$

Предложение 3. Для асимптотической эффективности последовательной прочедуры упорядочивания Бекхофера при любых $\boldsymbol{\theta} \in \Theta_{\Delta}$ верны соотношения:

(a) если $m=2$, mo

$$
\mathscr{E}(\boldsymbol{\theta}) \geqslant \frac{8 \Delta\left(\theta_{2}-\theta_{1}\right)}{m\left(\theta_{2}-\theta_{1}+\Delta\right)^{2}}
$$

(b) если $m \geqslant 3$, mо

$$
\mathscr{E}(\boldsymbol{\theta}) \geqslant \sum_{i=1}^{m-1} \frac{4 \Delta}{m\left(\theta_{i+1}-\theta_{i}+\Delta\right)^{2}} \min _{1 \leqslant i \leqslant m-1}\left(\theta_{i+1}-\theta_{i}\right) .
$$

Максимум асимптотической эффективности прочедурь при $m \geqslant 3$ допускает оценку

$$
\sup _{\boldsymbol{\theta} \in \Theta_{\Delta}} \mathscr{E}(\boldsymbol{\theta}) \geqslant \frac{m-1}{m}
$$

а при $m=2$ равен 1 . 
Д о к а з а т е л ь с т в о. Пусть $\varphi$ - процедура Бекхофера. Числитель в (4) оценивается путем последовательного применения предложения 1 и леммы 1 . Выражение для знаменателя в (4) получаем из (5). Производя теперь простые алгебраические операции, легко приходим к доказываемым неравенствам (6) и (7).

Выражение для оценки максимальной асимптотической эффективности получаем при $\boldsymbol{\theta}$ таком, что $\theta_{i+1}-\theta_{i}=\Delta, 1 \leqslant i \leqslant m-1$, - наименее благоприятная для упорядочивания ситуация. Предложение 3 доказано.

Интересно сравнить поведение максимума асимптотической эффективности процедуры упорядочивания Бекхофера и эффективность аналогичной процедуры отбора Бекхофера, полученной в работе [3]. Если при $m=2$ процедуры упорядочивания и отбора эффективны, то при $m \rightarrow \infty$ их поведение существенно различается: эффективность процедуры отбора Бекхофера монотонно убывает до 0.5, а эффективность процедуры упорядочивания, убывая при $m=3$ до значения 0.66 , в дальнейшем при росте $m$ возрастает до 1.

\section{СПИСОК ЛИТЕРАТУРЫ}

1. Володин И. Н. Нижние границы для среднего объема выборки и эффективность процедур статистического вывода. - Теория вероятн. и ее примен., 1979, т. 24, в. 1, c. $119-129$.

2. Володин И.Н. Нижние границы для среднего объема выборки в процедурах с управлением. - Теория вероятн. и ее примен., 1981, т. 26 , в. 3 , с. $630-631$.

3. Кареев И.А. Нижние границы для среднего объема выборки и эффективность процедур отбора. - Теория вероятн. и ее примен., 2012, т. 57, в. 2, с. 278-295.

4. Малютов М. Б. Нижние границы для средней длительности последовательно планируемых экспериментов.- Изв. вузов, сер. матем., 1983, № 11, с. 19-41.

5. Bechhofer R.E. A single-sample multiple decision procedure for ranking means of normal populations with known variances.- Ann. Math. Statist., 1954, v. 25, p. 1639.

6. Bechhofer R. E., Kiefer J., Sobel M. Sequential Identification and Ranking Procedures, with Special Reference to Koopman-Darmois Populations. Chicago: Univ. Chicago Press, 1968, 420 p.

7. Beirlant J., Dudewicz E. J., van der Meulen E. C. Complete statistical ranking of populations, with tables and applications. Departement Wiskunde: Mededelingen uit het Wiskundig Instituut Katholieke Universiteit Leuven, v. 145. Louvain: Defense Technical Information Center, 1981, 60 p.

8. Bishop T. A., Dudewicz E. J. Complete ranking of reliability-related distributions. IEEE Trans. Reliab., 1977, v. 26, № 5, p. 362-365. (Подробное изложение: Technical report № 114. Stanford: Stanford Univ., 1976.)

9. Galtchouk L. I., Maljutov M. B. One bound for the mean duration of sequential testing homogeneity. - MODA 4 - Advances in Model-Oriented Data Analysis (Spetses, Greece, 1995). Ed. by C. P. Kitsos. Heidelberg: Physica-Verlag, 1995, p. 49-56.

10. Schafer R.E., Rutemiller H.C. Some characteristics of a ranking procedure for population parameters based on chi-square statistics. - Technometrics, 1975, v. 17, № 3, p. 327-331.

11. Sobel M. J. Complete ranking procedures with appropriate loss functions. - Comm. Statist. Theory Methods, 1990, v. 19, № 12, p. 4525-4544.

Поступила в редакцию 10.II.2010

Исправленный вариант 11.IV.2011 